\title{
Pneumococcal spherical pneumonia multiply distributed in one lung
}

\author{
Y. Katsumura*, K. Shirakami*, S. Satoh**
}

Pneumococcal spherical pneumonia multiply distributed in one lung. Y. Katsumura, K. Shirakami, S. Satoh. @ ERS Journals Ltd 1997.

ABSTRACT: Spherical pneumonia, which is seen more frequently in children than in adults, is usually manifested as a solitary nodule. We report the case of a middleaged man with small spherical pneumonia, with multiple distribution in the right lung, caused by Streptococcus pneumoniae.

This type of pneumonia is very unusual and should be included in the differential diagnosis of multiple nodular densities of the lung. Eur Respir J 1997; 10: 2423-2424.

Spherical pneumonia simulating a pulmonary neoplasm is known to occur predominantly in children [1], but also occurs in adults [2]. In most reported cases, radiographic manifestation of this pneumonia is a solitary round nodule with or without hilar lymphadenopathy. This lesion is predominantly located in the posterior segment of the lower lobe [3]. We have recently seen an adult with multiple nodular densities, that were subsequently shown to be multiple spherical pneumonia caused by Streptococcus pneumoniae. This case demonstrated the relationship between pneumococci and this unusual type of pneumonia.
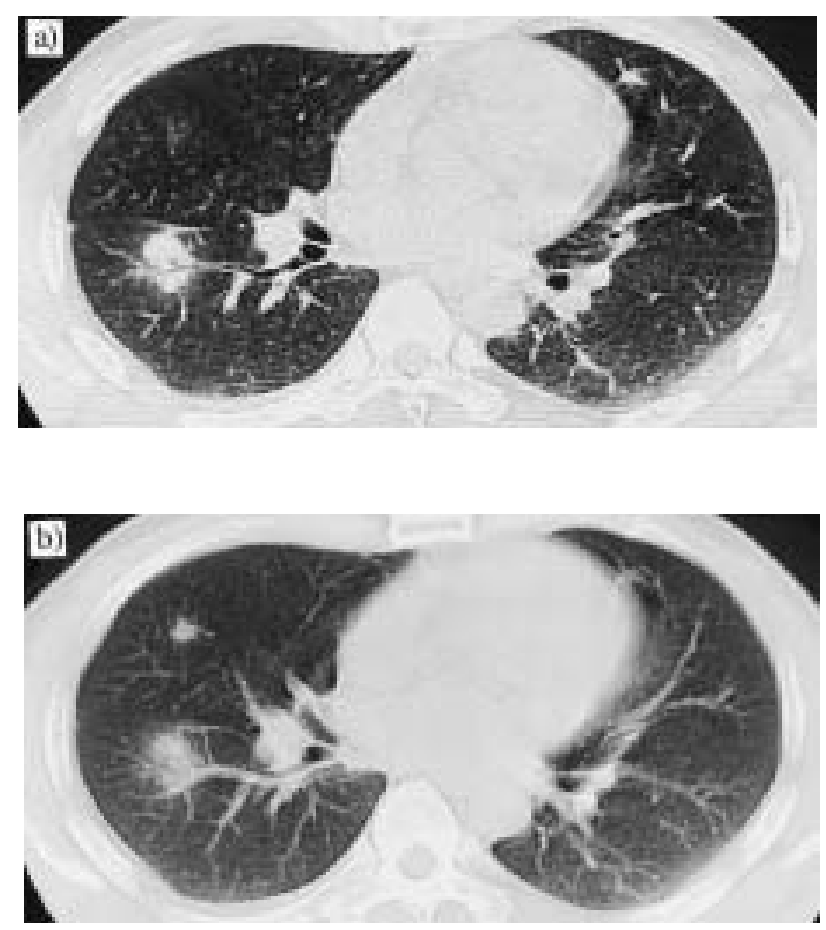
al effusion.
Depts of *Internal Medicine and **Radiology, Yokohama Asahi Chuo General Hospital, Kanagawa, Japan.

Correspondence: Y. Katsumura, Dept of Internal Medicine, Yokohama Asahi Chuo, General Hospital, 4-20-1 Wakabadai, Asahi-ku, Yokohama City 241, Japan

Keywords: Multiple, pneumococcal infection, spherical pneumonia

Received: March 201997

Accepted after revision June 171997

\section{Case report}

A $40 \mathrm{yr}$ old man was admitted to hospital with symptoms of high fever and right-sided chest pain. Fever developed 2 weeks prior to admission, when he also experienced dry cough and subsequently right pleuritic chest pain. There was no other significant past history.

On physical examination, the patient was a muscular man, with a temperature of $38.9^{\circ} \mathrm{C}$. The oropharynx was injected, and chest auscultation revealed diminished breath sounds, with no evidence of crackles at the right lung base. The remainder of the physical examination was unremarkable. Laboratory data showed an elevated white blood cell count $\left(10.1 \times 10^{9}\right.$ cells $\left.\cdot \mathrm{L}^{-1}\right)$ and $\mathrm{C}$ reactive protein $\left(27 \mathrm{mg} \cdot \mathrm{dL}^{-1}\right)$.

Chest radiographs revealed a spherical mass $(1.7 \times 1.8$ $\mathrm{cm})$ in the right middle lung area and two other nodular opacities in the right lower lung area. A computed tomography (CT) scan of the chest disclosed several nodular densities, $1-5 \mathrm{~cm}$ in diameter, in the right middle and lower lobes (fig. 1a-c). Thin-section images

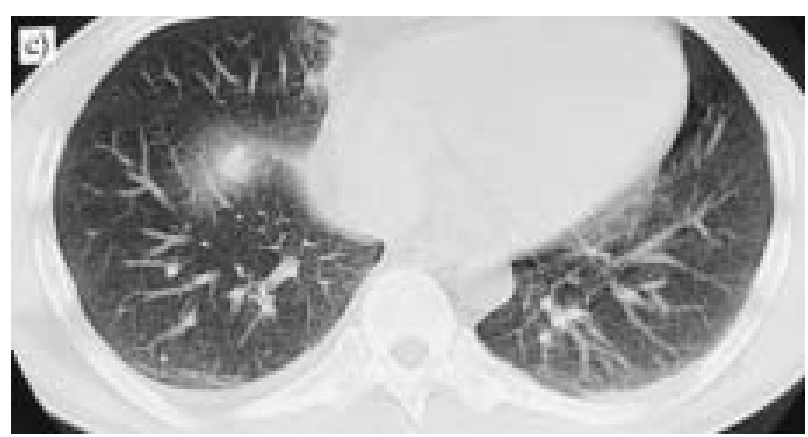

Fig. 1. - a) A thin-section image of a computed tomography (CT) scan of the chest showing a clear air-bronchogram in a nodule located in the superior segment of the right lower lobe. b) CT scan demonstrating a nodular density in the middle lobe and part of another nodule in the right lower lobe. c) CT scan showing a nodular opacity in the anterior segment of the right lower lobe. There is also a right pleur- 
disclosed a clear air-bronchogram in one of these nodules (fig. 1a). A right pleural effusion was also noted. A culture of sputum and bronchial excretion yielded abundant $S$. pneumoniae. A blood culture performed before administration of antibiotic treatment was negative.

The patient was treated with $2 \mathrm{~g}$ of panipenem-betamipron daily and his clinical condition then rapidly improved. A follow-up CT scan and chest radiographs taken 2 weeks after admission showed substantial reductions in the size of the nodular densities. The patient was discharged 3 weeks later. A CT scan taken 1 month after discharge showed only minimal residue.

\section{Discussion}

Spherical pneumonia is most commonly seen in children, but is not infrequent in adults [2, 4]. In most reported cases, the radiographic manifestation of spherical pneumonia is a solitary round nodule with or without hilar lymphadenopathy. This lesion is predominantly located in the posterior portions of the lung [3]. Although a case with bilateral spherical shadows of the chest has been reported [3], this type of pneumonia, manifested as small nodular densities with multiple distribution in the lung, is very unusual. Together with the clinical manifestations, the hazy margins of the spherical densities and air-bronchogram seen in one of the nodules suggested an inflammatory nature.

The most popular pathogen of spherical pneumonia both in adults and children is, as in this case, S. pneumoniae [3]. Other pathogens, including Klebsiella pneumoniae $[3,5]$, Haemophilus influenzae [6], and Mycobacterium tuberculosis $[4,5]$ have also been reported.

The mechanism by which spherical pneumonia occurs has been explained by the high affinity of pneumococci with the type II alveolar cell [7, 8]. Because of this affinity, the inflammatory process begins in the alveolar tissue [9] and spreads centrifugally through pores of Kohn and channels of Lambert, without circumbronchial relationship. Because no segmental boundaries exist in the alveolar tissue, the result is a spherical configuration [3-5]. This type of spread is in contrast to segmental pneumonia, in which an inflammation distributes through the bronchial system.
The cause of the occurrence of multiple pneumonia in a previously healthy man is uncertain. The pharyngeal mucosa has been thought to be the primary colonization site of pneumococci, and the bacteria spread down into the lower respiratory tract probably by aspiration $[8,10]$. In this case, repeated aspirations might have caused multiple foci of pneumonia. Although the blood culture was negative, both intrabronchial and haematogenic spreading of pneumococci could be possible mechanisms for the multiplicity of infection.

Finally, in patients with multiple nodular densities, particularly when they have hazy margins and airbronchograms, multiple spherical pneumonia should be included in the differential diagnosis in order to avoid unnecessary diagnostic procedures.

\section{References}

1. Talner LB. Pleuropulmonary pseudotumors in childhood. Am J Roentgenol 1967; 100: 208-213.

2. Hershey CO, Panaro V. Round pneumonia in adults. Arch Intern Med 1988; 148: 1155-1157.

3. Rose RW, Ward BH. Spherical pneumonias in children simulating pulmonary and mediastinal masses. Radiology 1973; 106: 179-182.

4. Greenfield H, Gyepes MT. Oval-shaped consolidations simulating new growth of the lung. Am J Roentgenol 1964; 91: 125-131.

5. Fraser RG, Wortzman G. Acute pneumococcal lobar pneumonia: the significance of non-segmental distribution. J Can Assoc Radiol 1959; 10: 37-46.

6. Sproul JM. Spherical pneumonia due to Hemophilus influenzae: a definitive study by transtracheal aspiration. Am Rev Respir Dis 1969; 100: 67-69.

7. Cundell DR, Tuomanen EI. Receptor specificity of adherence of Streptococcus pneumoniae to human type-II pneumocytes and vascular endothelial cells in vitro. Microb Pathog 1994; 17: 361-374.

8. Tuomanen EI, Austrian R, Masure HR. Pathogenesis of pneumococcal infection. $N$ Engl J Med 1995; 332: $1280-1284$

9. Robertson OH, Coggeshall LT, Terrell EE. Experimental pneumococcus lobar pneumonia in the dog: III. pathogenesis. J Clin Invest 1933; 12: 467-493.

10. Weiser JN, Austrian R, Sreenivasan PK, Masure HR. Phase variation in pneumococcal opacity: relationship between colonial morphology and nasopharyngeal colonization. Infect Immun 1994; 62: 2582-2589. 